Estudio de Caso

\title{
Hepatitis autoinmune en un paciente con hepatitis B crónica, a propósito de un caso
}

\section{María Teresa Domínguez Plaja ${ }^{1}$, Luis Gustavo Celis ${ }^{2}$, Andrés Felipe Posada López ${ }^{3}$}

\author{
${ }^{1}$ Estudiante Facultad de Medicina Universidad de La Sabana. \\ ${ }^{2}$ Docente e Investigador Facultad de Medicina Universidad de La Sabana \\ ${ }^{3}$ Médico general. Residente Medicina Interna Facultad de Medicina Universidad de La Sabana.
}

Correspondencia: maria.dominguez@unisabana.edu.co

Recibido: 18/04/2013 Aceptado: 19/05/2013

\section{Resumen}

La hepatitis es una enfermedad viral que azota a gran parte de la población mundial y cuya etiología es multifactorial, por lo que se ha clasificado a fin de hacer más conveniente su estudio de la A a la E, separándolas según sus características. Existe además la hepatitis autoinmune (HAI), entidad de etiología no conocida que gana mayor prevalencia e incidencia cada año. Se cuenta con pocos reportes en la literatura que relacionen la presentación de una hepatitis viral más una hepatitis autoinmune en un mismo paciente. Sin embargo, algunos estudios han comprobado que dicha relación es una posibilidad; lo que dificulta su orientación diagnóstica, terapéutica, tratamiento y pronóstico. Se presenta un caso de hepatitis B crónica en la que se superpuso una hepatitis autoinmune, a fin de realizar una revisión del tema y sus dificultades diagnósticas.

Palabras clave: hepatitis autoinmune, hepatitis B crónica, autoanticuerpos

\section{Topic Review: Autoimmune hepatitis in a patient with chronic hepatitis B. A case reportAbstract}

\begin{abstract}
Hepatitis is a viral disease that affects a large portion of the world's population, which has a multifactorial etiology, thus to facilitate its study has been classified from A to E according with the characteristic. Additionally to viral hepatitis also occurs the autoimmune hepatitis, a disease of unknown etiology of which prevalence and incidence are increasing every year. There is few reports in the literature that describe the possibility of finding viral hepatitis and autoimmune hepatitis in one patient at the same time. Nevertheless some studies have indicated that there is possible correlation between them, situation that make difficult its diagnosis, therapeutic approach, treatment and prognosis. A case of chronic B hepatitis with overlapped autoimmune hepatitis is reported aiming to, get a topic review and diagnostic difficulties.
\end{abstract}

Key words: autoimmune hepatitis, chronic B hepatitis, auto-antibodies 


\section{Introducción}

La hepatitis B es una enfermedad viral infectocontagiosa que se produce principalmente por transmisión sanguínea o exposición sexual directa con una persona infectada con Hepadnavirus, familia del agente causal (1-3). La capacidad lesiva del virus es despreciable, pero se sabe que la respuesta inmune del huésped a los antígenos virales es la que produce la lesión hepatocelular propiamente dicha. Aproximadamente un tercio de la población mundial tiene evidencia de infección previa o actual con el virus causal y entre $350 \mathrm{y}$ 400 millones de personas positivos para el antígeno de superficie (3). La hepatitis B crónica se desarrolla en un proceso largo en el que el virus de forma primaria se replica constantemente dentro del organismo haciéndose altamente contagioso, para posteriormente disminuir la tasa de replicación paulatinamente, pero finalmente convirtiéndose en virus activo.

En fases iniciales y activas, los títulos de $\mathrm{HBsAg}$ (antígeno de superficie), anti $\mathrm{HBc}$ total y anti $\mathrm{HBc}-$ IgM son positivos con niveles normales o bajos de aminotransferasas y escasa inflamación del tejido hepático, siendo factible que la respuesta inmune permita la adecuada eliminación de la enfermedad en muchos casos (4). A pesar de esto, para considerar inactivo un portador del virus debe hacerse seguimiento al menos cuatro meses de los niveles de las aminotransferasas, las cuales deben estar dentro de los parámetros normales. No obstante, si los cambios en el funcionamiento y la estructura hepática se van conformando en el tiempo, la enfermedad avanza y se expresa principalmente en la alteración de las aminotransferasas. La cronicidad de la enfermedad se denota cuando hay presente anti HBcIgM y HBsAg positivo (4).

La hepatitis autoinmune (HAI), es una enfermedad crónica y progresiva de etiología desconocida (5). Para su estudio se han planteado diferentes hipótesis diagnósticas con el objetivo de orientar la terapéutica de la patología. Pero fue hasta 1966 que la relación con los anticuerpos anti-músculo liso fue observada por Whittingham conociéndose desde entonces como hepatitis crónica activa autoinmune (5). Según Orts Costa y Colbs se considera como una enfermedad necro-inflamatoria del hígado, infrecuente, usualmente crónica y progresiva, de etiología no suficientemente conocida que se caracteriza por la presencia de alteraciones inmunológicas entre las que se encuentran la hipergammaglobulinemia y la presencia de autoanticuerpos, con una buena respuesta al tratamiento con inmunosupresores y mayor prevalencia en mujeres, en especial jóvenes, y que característicamente tiene períodos de remisión. No existe un marcador patognomónico de esta enfermedad (6).

La superposición clínica de hepatitis B crónica y hepatitis autoinmune no se ha referenciado con anterioridad en nuestro medio. Sin embargo existen reportes que relacionan ambas enfermedades en países desarrollados. No obstante, las dificultades diagnósticas y el desconocimiento del solapamiento de estas dos patologías hacen menester la revisión basada en este caso.

\section{Presentación del caso}

Mujer de 58 ańos con antecedentes de falla cardíaca, enfermedad de Parkinson e hipertensión arterial, que consulta por cuadro clínico de un mes de evolución consistente en edema de miembros inferiores grado I que progresó paulatinamente hasta ascitis a tensión. Como síntomas asociados presentó varios episodios eméticos post-prandiales de contenido biliar acompañado de epigastralgia, disnea de pequeños esfuerzos y tos no productiva. Al examen físico, paciente con estabilidad hemodinámica, escleras ictéricas. Ruidos cardiacos rítmicos con soplo telesistólico en foco pulmonar y aórtico, murmullo vesicular disminuido con estertores en ambos campos pulmonares, ascitis severa, reflejo hepato-yugular positivo, edema grado 3 en miembros inferiores, llenado capilar de 5 segundos. Se realiza ecografía abdominal total que demuestran cambios en ecogenicidad y tamaño hepáticos, distensión en vesícula con presencia de barro biliar, líquido en cavidad abdominal y cavidad pleural. Se decide realizar paracentesis diagnóstica y 
evacuatoria con gradiente de albúmina para descartar peritonitis bacteriana espontánea. Se toman; marcadores de hepatitis B consistentes en antígeno de superficie para hepatitis B (HBsAg), anti-core para virus de la hepatitis $\mathrm{B}$, antígeno $\mathrm{E}$ para virus de hepatitis $\mathrm{B}(\mathrm{AgEVHB})$, anticuerpo para el virus de la hepatitis (AcVHC), ferritina, transferrina, anticuerpos antinucleares (ANAS), anticuerpos anti-musculo liso (ASMA), anticuerpos anti-mitocondriales (AMA), alfa 1 anti-tripsina, ceruloplasmina y electrolitos. Se obtienen resultados de albúmina sérica de $1.09 \mathrm{mg} /$ $\mathrm{dL}$, bilirrubina total $2.72 \mathrm{mg} / \mathrm{dL}$, bilirrubina directa $1.35 \mathrm{mg} / \mathrm{dL}$, bilirrubina indirecta $1.37 \mathrm{mg} / \mathrm{dL}$, AST 80 U/L, ALT 16 U/L, PT 19.6, PTT 66.5 y un INR de 1.78, Figura 1, radiografía de tórax con hallazgos de falla cardíaca con redistribución de flujo e hipertensión pulmonar acompañado de opacidades en ambos hemitórax con predominio derecho con características de derrame pleural. HBsAg positivos con valor 7156, anti core reactivo mayor 0.05 , anti HCV negativo (Agosto 2012), por lo que se diagnostica enfermedad hepática crónica secundaria a virus de Hepatitis B, solicitándose carga viral. Se inicia tratamiento con Entecavir a dosis basadas en la evidencia para estas patologías.

Se decide realizar segunda ecografía abdominopélvica aumento de ascitis la cual se torna a tensión, que demuestra cambios congruentes con cirrosis hepática y signos de hipertensión portal asociada.

Tabla 1. Pruebas confirmatorias de enfermedad hepática realizadas a la paciente.

\section{Nombre de la Prueba}

\section{Resultados del caso}

Anticuerpos centrales totales Hepatitis B (Anti core $\mathrm{Hb}$ )

Mayor 0.005 Reactivo

Antígeno de superficie Hepatitis B (Ag HbS)

7156 Reactivo

Hepatitis C anticuerpo

No reactivo

Anticuerpo antinucleares (ANAS)

Mayor a $1 / 320$

Anticuerpos anti-músculo liso (AMAS)
Se reciben reporte de ANAS y ASMA reactivos 1/320 sugestivo de mitocondrial, sustrato de ANAS HEP2 Tabla 1, decidiéndose tomar biopsia hepática por la presunción diagnostica de hepatitis autoinmune superpuesta a hepatitis B crónica, con aras de descartar un epifenómeno asociado a la infección por el virus de la hepatitis $\mathrm{B} y$, adicionalmente, se inicia tratamiento con Esteroide por el diagnóstico de hepatitis autoinmune superpuesta a hepatitis B crónica. A pesar de tratamiento integral para falla cardiaca la paciente persiste con edema generalizado y sobrecarga de volumen, sin mejoría de signos de congestión. Se practica toracentesis terapéutica, drenándose $500 \mathrm{~mL}$ de líquido claro, el cual al estudiarse reporta ser un exudado linfocitario por criterios de Light, sin evidencia de gérmenes, considerándose asociado a compromiso por su enfermedad autoinmune.

La paciente presenta deterioro clínico manifestado por dificultad respiratoria progresiva que la lleva a falla ventilatoria aguda, por lo que se inicia ventilación no invasiva. Se intenta practicar paracentesis, la cual no es efectiva por no lograrse extraer líquido abdominal. Se realiza ecografía de pericardio, pleura y tórax que reporta atelectasia compresiva y derrame pleural tabicado con septos de grosor importante, con desplazamiento del cardiomediastino hacia la izquierda. Presenta deterioro de la función hepática con valores de análisis de albúmina 2.2 $\mathrm{mg} / \mathrm{dL}$, bilirrubina directa $3.36 \mathrm{mg} / \mathrm{dL}$, bilirrubina indirecta $4.43 \mathrm{mg} / \mathrm{dL}$, bilirrubina total $7.53 \mathrm{mg} / \mathrm{dL}$, AST $125 \mathrm{U} / \mathrm{L}$, ALT $95 \mathrm{U} / \mathrm{L}$, Tabla 2, fosfatasa alcalina $124 \mathrm{LDH} 338$, proteínas totales $6.2 \mathrm{~g} / \mathrm{dL}$, PT 40.8, PTT 270 y un INR de 4.18, Septiembre 2012, Figura 1. 
Tabla 2. Comparativo pruebas de función hepática. AST: Aspartato amino-transferasa; ALT: Alanino amino-transferasa.

\begin{tabular}{lll}
\hline \multicolumn{1}{|c}{$\begin{array}{c}\text { Prueba } \\
\text { Solicitada }\end{array}$} & \multicolumn{1}{c}{$\begin{array}{c}\text { Resultados } \\
\text { Agosto } \\
\mathbf{2 0 1 2}\end{array}$} & $\begin{array}{c}\text { Resultados } \\
\text { Septiembre } \\
\mathbf{2 0 1 2}\end{array}$ \\
\hline Albúmina & $1.09 \mathrm{mg} / \mathrm{dL}$ & $2.2 \mathrm{mg} / \mathrm{dL}$ \\
\hline $\begin{array}{l}\text { Bilirrubina } \\
\text { indirecta }\end{array}$ & $1.37 \mathrm{mg} 7 \mathrm{dL}$ & $4.43 \mathrm{md} / \mathrm{dL}$ \\
\hline $\begin{array}{l}\text { Bilirrubina } \\
\text { total }\end{array}$ & $2.72 \mathrm{mg} / \mathrm{dL}$ & $7.53 \mathrm{mg} / \mathrm{dL}$ \\
\hline AST & $80 \mathrm{U} / \mathrm{L}$ & $125 \mathrm{U} / \mathrm{L}$ \\
\hline ALT & $16 \mathrm{U} / \mathrm{L}$ & $95 \mathrm{U} / \mathrm{L}$ \\
\hline
\end{tabular}

La paciente persiste con deterioro clínico pese a las medidas instauradas, presentando posteriormente paro cardio-respiratorio y fallecimiento, por lo que no se pudo realizar biopsia hepática.

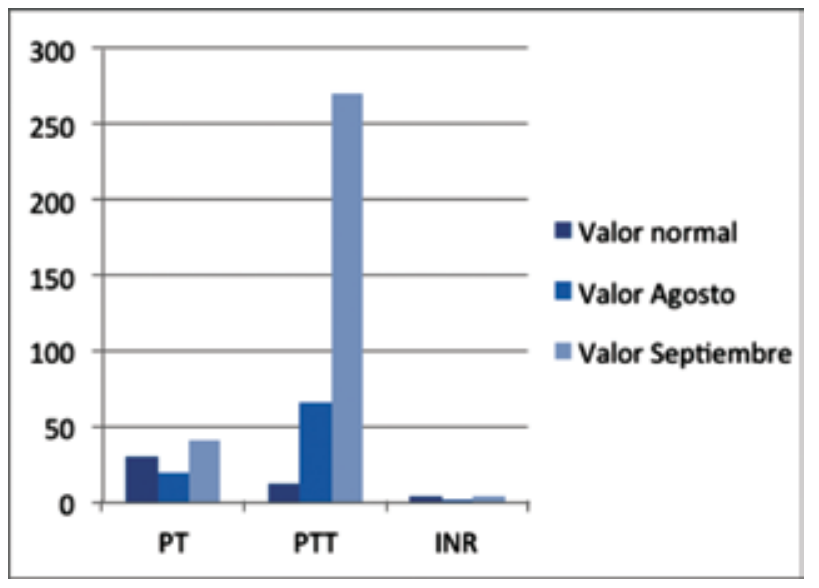

Figura 1. Pruebas coagulación

\section{Discusión}

La HAI es una enfermedad hepática necroinflamatoria crónica caracterizada por la presencia de autoanticuerpos circulantes, hipergammaglobulinemia con valores elevados de inmunoglobulina $\mathrm{G}(\mathrm{IgG})$ y una lesión histológica necroinflamatoria con hepatitis de interfase e infiltrado inflamatorio linfoplasmocitario $(6,7)$.

El síndrome de superposición entre hepatitis $\mathrm{B}$ y hepatitis autoinmune debe ser definido cuando existan en un mismo paciente la expresión de las características clínicas, bioquímicas e histológicas de ambas enfermedades (8). Su correcto diagnóstico tiene implicaciones clínicas relevantes en cuanto a las consecuencias pronosticas y de tratamiento (9).

La posibilidad de HAI aumenta al encontrar persistentemente las aminotransferasas elevadas $\mathrm{y}$ usualmente acompañada por gammaglobulinemia, el $25 \%$ son asintomáticas al diagnóstico. El $40 \%$ se puede presentar como hepatitis aguda con ictericia, anorexia, náusea y síntomas similares a la influenza (10).

La HAI se ha categorizado en dos subtipos; Tipo 1 que está asociada con la presencia de anticuerpos antinucleares (ANAs) y anticuerpos anti-músculo liso (ASMA) en la cual se encuentra el $75 \%$ de los pacientes y la tipo 2 que cuenta con la presencia de anticuerpos anti-microsomales higado riñón 1 (LKM-1) o anticuerpos anti-citosólico hepático (LC-1). En la mayoría de centros el $10-25 \%$ de los pacientes tendrán títulos indetectables de anticuerpos y han sido diagnosticados previamente como hepatitis crónica criptogénica. El díagnóstico se debe hacer sobre la base de los criterios diagnósticos de la IAIHG. Las pruebas bioquímicas e inmunológicas son insuficientes para definir el diagnóstico de IAH. Es importante la realización de biopsia hepática para apoyo del diagnóstico y adicionalmente información pronóstica (11).

En presencia de HAI, se debe descartar la actividad replicativa vírica, aunque ello implica una mayor dificultad diagnóstica ya que, como se ha comentado, la presencia de $\mathrm{HBsAg}$ positivo es criterio que penaliza en los sistemas de puntuación de los criterios diagnósticos de HAI (12).

Otro punto a destacar es si la infección crónica por el VHB pudiera tener relación con un posible factor desencadenante del proceso inmunológico, hecho referenciado en la literatura médica (13). La base etiopatogénica barajada es la del mimetismo molecular por la que un antígeno externo genera una respuesta inmunológica cruzada frente a un antígeno propio, al compartir secuencias de 
aminoácidos en su cadena peptídica. Los autoanticuerpos nucleares y musculo liso son comunes en infección por el virus de la hepatitis B. Aunque los mecanismos son inciertos los linfocitos $\mathrm{T}$ CD4 ayudadores reaccionan contra el AgcHB y $\mathrm{AgSHB}$ en la superficie expuesta de los hepatocitos infectados durante la infección por VHB llevando a linfocitos $\mathrm{B}$ específicos para antígenos hepatocelulares normales conduciendo a la producción de autoanticuerpos específicos contra el hepatocito lo que favorece a reacción citotóxica contra el hepatocito infectado lo cual puede causar hepatitis autoinmune. Los autoanticuerpos nucleares y musculo liso son comunes en infección por el virus de la hepatitis B. Su origen es porque la DNA polimerasa del VHB comparte 7-9 secuencias de aminoácidos con los anticuerpos nucleares y proteínas del músculo liso (miosina y caldemosina) (14). Los ANA y ASMA están presentes en el $20-40 \%$ de estos pacientes principalmente en infección por virus de la hepatitis C (15).

En un estudio de 25 pacientes con hepatitis autoinmune 20 pacientes $(80 \%)$ tenían anticuerpos positivos para virus de hepatitis $\mathrm{C}$ a diferencia de hepatitis B que solo lo tenían 5 pacientes (20\%). Basados en los sistemas de puntuación ninguno de los pacientes fue diagnosticado como hepatitis autoinmune definitiva. 18 pacientes $(72 \%)$ fueron diagnosticados como probable y el 7 restante $(28 \%)$ como diagnóstico de HAI probable. Sin embargo los sistemas de puntuación no son útiles solos para el diagnóstico. Este se debe basar en el perfil serológico, hallazgos histológicos, sistemas de puntuación, respuesta al tratamiento y desenlaces (16). El diagnóstico debe hacerse en pacientes con signos clínicos compatibles, síntomas y alteraciones analíticas. Ayudas de laboratorio compatibles y anormalidades histológicas que incluyen pruebas bioquímicas hepáticas anormales, un aumento del total de IgG o niveles de gammaglobulina, marcadores serológicos (ANA, ASMA, anti-LKM-1 o anti-LC1), y hepatitis interfase. Deben excluirse otras condiciones que pueden causar hepatitis crónica (9).
Es importante tener en cuenta en pacientes con VHB la necesidad de tratamiento antiviral profiláctico cuando se va a precisar un tratamiento inmunosupresor, como fue descrito en nuestra paciente (3).

Las terapias para hepatitis B crónica buscan disminuir la disfunción hepática, pero también garantizar que la viremia sea menor. Tratamientos con Interferón alfa en hepatitis B crónica han reportado disminuir el riesgo de carcinoma hepatocelular, una de las complicaciones más alarmantes de la enfermedad. Sin embargo sus efectos adversos hacen difícil su uso en muchos pacientes, además de los costos. Hasta el momento, el uso de antivirales sigue siendo el pilar fundamental de la terapéutica. Medicamentos como la Lamivudina, Entecavir, o Tenofovir, constituyen herramientas muy útiles por su efecto análogo de nucleótidos de acción directa en la transcriptasa reversa, que modifica la replicación viral y, con menor evidencia, su efecto inmunomodulador, aspecto que sigue en estudios clínicos (3).

En la hepatitis autoinmune, la supervivencia a los cinco años en pacientes sin tratamiento es de aproximadamente $25 \%$ vs $80 \%$ de los que son tratados con corticosteroides. La Asociación Americana para el Estudio de Enfermedades Hepáticas (AASLD) recomiendan Prednisona a dosis inicial de $30 \mathrm{mg} /$ día combinado con $1.2 \mathrm{mg} /$ kg/día de Azatioprina o en monoterapia: Prednisona a una dosis inicial de 40-60 mg/día en adultos (6).

El uso de terapias combinadas que incluyan medicamentos para tratar ambas enfermedades no se ha descrito previamente, pero al parecer es la conducta más efectiva en casos como este. No obstante, es vital no olvidar soportar las posibles complicaciones por sobrecarga, que son tan frecuentes en éstos pacientes, tal y como se ha visto en el caso descrito, puesto que empeoran el curso clínico y terapéutico de la enfermedad, dificultando el manejo de la misma. 
El seguimiento de las pruebas de función hepática es una herramienta que permite identificar el impacto del tratamiento implementado en el estado del paciente. Niveles altos persistentes de aminotransferasas indicarán que la lesión hepática persiste a pesar de las medidas tomadas.

Dada la falta de referencias existentes de casos de hepatitis autoinmune sobrepuesta a hepatitis B crónica, existe mucho aún por investigar en éste tópico, por lo que la decisión y enfoque clínico dependen de manera directa del apoyo multidisciplinario de salud, y el diagnóstico certero sólo será posible si se evidencian en un mismo paciente los hallazgos de laboratorio característicos para cada patología ya descritos. De no ser así, será mejor contemplar otras posibilidades diagnósticas que incluyan superposiciones de hepatitis virales o complicaciones hepáticas crónicas como cirrosis o carcinoma hepatocelular, e incluso lesión hepática medicamentosa inducida por tratamientos para hepatitis.

\section{Referencias}

1. Ganem D, Prince AM. Hepatitis B Virus Infection - Natural History and Clinical Consequences. N Engl J Med. 2004 Mar 11;350(11):1118-1129.

2. Czaja AJ, Manns MP. Advances in the Diagnosis, Pathogenesis, and Management of Autoimmune Hepatitis. Gastroenterology 2010; 139: 58 -72.

3. EASL clinical practice guidelines. Management of chronic hepatitis B virus infection. J Hepatol. 2012;57:167-185

4. Servoss JC, Friedman Serologic and molecular diagnosis of hepatitis B virus. Infect Dis Clin North Am. 2006 Mar;20(1):47-61.
5. Krawitt EL. Autoimmune Hepatitis. N Engl J Med 2006; 354: 54-66.

6. Manns MP, Czaja AJ, Gorham JD, Krawitt EL, Mieli-Vergani G, Vergani D, Vierling JM; American Association for the Study of Liver Diseases. Diagnosis and management of autoimmune hepatitis. Hepatology. 2010 Jun;51(6):2193-2213.

7. Núnez Martínez Ó, et al. Evolución de cirrosis biliar primaria a síndrome de solapamiento con hepatitis autoinmune en paciente con hepatitis B crónica. Gastroenterol Hepatol. 2012. Oct 17. pii: S0210-5705(12)00254-3

8. Woodward J, Neuberger J. Autoimmune overlap syndromes. Hepatology. 2001;33:994-1002.

9. Boberg KM, Chapman RW, Hirschfield GM, Lohse AW, Manns MP, Schrumpf E. Overlap syndromes: the international autoimmune hepatitis group (IAIHG) position statement on a controversial issue. J Hepatol. 2011;54:374-85

10. Rust C, Beuers U. Overlap syndromes among autoimmune liver diseases. World J Gastroenterol 2008 June 7; 14(21): 3368-3373.

11. Gleeson D, Heneghan MA. British Society of Gastroenterology (BSG) guidelines for management of autoimmune hepatitis. Gut 2011 Dec;60(12):1611-29

12. Hennes EM, Zeniya M, Czaja AJ, Parés A, Dalekos GN, Krawitt EL, et al. Simplified criteria for the diagnosis of autoimmune hepatitis. Hepatology. 2008;48:169---76.

13. Núnez Martínez O, Clemente Ricote G. Hepatitis autoinmune. Etiopatogenia. GH Continuada. 2005;4:201-6.

14. Gregorio GV, Choudhuri K, Ma Y, Vegnente A, Mieli-Vergani G, Vergani D. Mimicry between the hepatitis B virus DNA polymerase and the antigenic targets of nuclear and smooth muscle antibodies in chronic hepatitis B virus infection. J Immunol. 1999; 162:1802-10

15. Nobili V et al. Co-occurrence of chronic hepatitis B virus infection and autoimmune hepatitis in a young Senegalese girl. European Journal of Gastroenterology \& Hepatology,2006;18( 8)

16. Efe C, Wahlin S, Ozaslan E, Purnak T, Muratori L, Quarneti C, Tatar G, Simsek H, Muratori P, Schiano TD. Diagnostic difficulties, therapeutic strategiesand performance of scoring systems in patients with autoimmune hepatitis and concurrent hepatitis B/C. Scand J Gastroenterol. 2013; 48(4):504-8. 